\title{
INTEGRATING PSYCHEDELIC EXPERIENCES UTILIZING THE INTERNAL FAMILY SYSTEMS THERAPEUTIC MODEL
}

\author{
NANCY L. MORGAN, M.S., PhD. \\ https://doi.org/10.37602/IJSSMR.2020.3417
}

\begin{abstract}
This paper introduces the potential benefits of utilizing an innovative, evidence-based framework to support clients' integration of their psychedelic experiences, while also presenting personal views and cautionary feedback offered by a sampling of practitioners, authors, and laypeople that have directly utilized psychedelics, and/or are working with clients utilizing psychedelics. The opportunity to employ a framework that shares in common with psychedelic experiences essentials such as an awareness of oneself as greater than the sum of one's parts, the transformational qualities of embodied experiences, and a nonpathologizing perspective is seen as an ideal in the growing field of psychedelic-assisted psychotherapies.
\end{abstract}

Keywords: Internal Family Systems, Psychedelic, Integration

"Do I contradict myself? Very well, then, I contradict myself; I am large -- I contain multitudes."

Walt Whitman

\section{INTRODUCTION}

Over the past twenty years, an increasing number of sanctioned research studies have provided considerable insight into the beneficial uses of psychedelics for treating conditions such as Post-Traumatic Stress Disorder (PTSD) and the extreme anxiety that often arises following news that a client has been diagnosed with terminal cancer. These studies' outcomes are appearing increasingly more often in the mainstream press and generating a new wave of clinical therapy clients eager to explore the utility of psychedelics in their own lives. It is becoming more common for therapists to be asked by clients how psychedelics, such as ketamine, a legal, FDA- approved medication shown to have antidepressant benefits, might be utilized in therapy to bring clients relief from suffering, offer greater insights, and enhance perspective into their own lives.

Thus far, only one article (Sloshower, et al., 2020) has proposed a therapeutic framework for therapists to utilize with clients to aid in the integration process following a client's psychedelic experience: Acceptance and Commitment Therapy (ACT). This paper offers another evidence-based therapeutic framework: the Internal Family Systems (IFS) therapeutic model. 


\section{International Journal of Social Sciences and Management Review}

The aim of the current paper is to highlight the unique elements of the evidence-based, psychospiritual framework: Internal Family Systems (IFS) therapeutic model. The Internal Family Systems model elegantly supports the integration of psychedelic experiences, is nonpathologizing, views that we are all a multiplicity rather than comprised of a mono-mind, and introduces into the field of psychology that the client's own Self-to-part relationship forms the secure attachment enabling clients' healing. Per IFS, the qualities of a therapist best facilitate a client's Self-to-part relationship include what is referred to as the "8 C's:" Clarity, Calm,

Curiosity, Compassion, Confidence, Connectedness, Creativity, and Courage. These qualities are among those identified in the scant literature reporting on the qualities identified by clients as most desired in a therapist.

Ward \& Morgan (2017) reported on qualities clients most desired in a therapist utilizing clients' own words, rather than those of therapists. Inquiries into clients' preferences at the outset of therapy embodies a client-centred approach, one that endorses therapists' endorsing those innate qualities that enhance the quality of their therapeutic relationships with clients. Therapy has been defined as the ability to create a rapport with a client (Mosey, 1981 as cited in Palmadottir, 2006). The relationship between a client and a therapist is incredibly important in ensuring the success of the therapy (Levitt, Butler \& Hill, 2006; Littauer, Sexton \& Wynn, 2005).

In a review of therapist characteristics and techniques positively impacting the therapeutic alliance, Ackerman and Hilsenroth (2003) found that therapists' personal attributes, such as being respectful, warm, interested, and open, enhanced the therapeutic alliance. Lawlor, 2016; Owen, et al, (2016) cited empathy, genuineness, competence, and trustworthiness as contributing to client satisfaction with therapy. Littauer, et al, (2005) found that clients wanted their therapist to be warm, responsive, calm and understanding.

Set and Setting

The importance of set and setting have been emphasized in all research studies involving psychedelic medicines. Set references one's mindset and setting refers to the physical and social environment in which the user has the psychedelic experience. The setting must feel safe and supportive whenever working with psychedelic medicines as they often present clients with memories long exiled, sometimes for years, sometimes decades.

Of lesser focus has been the importance of how best clients can integrate their psychedelic experiences into their day-to-day lives. Too often clients are incited to engage in sanctioned and/or unsanctioned psychedelic experiences that forego the importance of providing integrative supports following a psychedelic experience. Ketamine infusion clinics have been opening offering no therapeutic supports of any sort, and one wonders if it isn't just a matter of time before this practice will result in grave harm, or worse, to a client.

The Importance of Integration

Three questions were posed to individuals whose work and experiences are helping to define the art and science of integration: Julie Megler, MSN, NP-BC, a board-certified nurse practitioner in psychiatry and family medicine; Rachel Harris, $\mathrm{PhD}$, author of Listening to 


\section{International Journal of Social Sciences and Management Review}

Ayahuasca, Internal Family Systems developer, Richard C. Schwartz, PhD, and Allison Walmsley, a subject in the MAPS Phase II MDMA study.

The Three Questions:

1. How do you define integration?

2. How important is integration from your perspective?

3. Do you have any concerns relative to how integration is or is not being incorporated into healing with psychedelic substances?

Julie Meghler, MSN, NP-PC (email communication November 18, 2019)

Question \#1: How do you define Integration?

Integration refers to the process by which the material accessed and insights gained in an entheogenic experience are incorporated over time into one's life in a way that benefits the individual and their community.

Question \#2: How important is Integration in the healing process, with or without psychedelics?

Critical! I like to tell clients that integration becomes a way of life, rather than something you complete and move on to. We must bring integration into all aspects of our lives. What the psychedelic journey accomplishes is minimal without us then integrating it into our lives. I could really elaborate here but will keep it to that for now.

Question \#3: Have you any concerns regarding how Integration is, or is not, currently being integrated into the use of psychedelic substances?

I think the MAPS protocol does a good job of incorporating integration into the protocol. However, if we look at man ketamine clinics there is minimal integration happening. If we are incorporating the discussion of how well integration is being helped internationally or in underground settings, I would also say insufficient attention is being put on it.

Rachel Harris, PhD (email communication November 18, 2019)

Question \#1: How do you define Integration?

I think of integration from a psychotherapy point of view even as it applies to psychedelics. I find it helpful to think in terms of integrating the personality: uniting the

"affective, cognitive, behavioural, and physiological systems within a person". from the wiki. I would add spiritually as well. Also, I think Dan Siegel's concept of coherence is relevant.

Richard Schwartz, PhD (email communication November 29, 2019) 


\section{International Journal of Social Sciences and Management Review}

Question \#1: How do you define Integration?

Integration is the harmonious, collaborative and loving operation of human systems at any level.

Question \#2: How important is Integration in the healing process, with or without psychedelics?

The German derivation of the word heal is to make whole and integration is central to making people or systems of people harmoniously whole.

Question \#3: Have you any concerns regarding how Integration is, or is not, currently being integrated into the use of psychedelic substances?

I have huge concerns about this issue because without proper guidance psychedelic experiences can create the opposite of integration- polarization, exiling, and psychosis-like disintegration of psychological systems. To prevent such experiences and to promote integration, guides need an ecologically sensitive map of the intrapsychic territory that can anticipate and calm fearful and backlash reactions to the massive shifts in that territory that psychedelic experiences can create. The guide must know how to maintain Self- energy, holding qualities like calm, compassion, confidence, clarity and connectedness and keep the space around the journeyer safe from psychic entities that can enter when they are out of their bodies. Guides must also be able to help journeyers approach the parts of them that spontaneously arise with curiosity and compassion which means they need an understanding that all parts are valuable regardless of how extremely they present.

Alison Walmsley (email communication December 2, 2019):

Question \#1: How do you define Integration?

The topic of integration is deep and complex; thank you for welcoming a diverse group into the discussion. My expanding understanding of integration is that it involves every aspect of our lived human experience. Yet, how we navigate the path forward feels deeply personal but also collective.

I immersed myself in the IFS/Tibetan Buddhism training last weekend. A beautiful reawakening of my healing process began again, through learning from these great teachers. I am in awe of the intersection of these two practices, and that they found each other. I am so excited to learn more. Any additional resources you can recommend would be greatly appreciated.

MDMA with therapy does a beautiful job of allowing parts to emerge and opening the access points of love, compassion, and trust. I think most MAPS participants would leave the study without a framework for continued work with parts, and the deepening mode would be cut short due to the nature of the study limiting integration sessions 


\section{International Journal of Social Sciences and Management Review}

Volume: 03, Issue: 04 "July - August 2020"

ISSN 2582-0176

and ultimately finishing within just three short months. I can now see how this happened with my process.

My definition of integration is multidimensional: Content - Deepening - Change. Content - It involves working with the content and material which emerged in the psychedelic session to process the experience, insights, and wounds which opened in the session. It necessitates a safe environment in which our stories are heard and held with compassion.

Deepening - It involves a deepening or watering of seeds sown during the psychedelic session through practices, and orienting ourselves to the possibility of a different future.

Change - Ultimately, integration may ask us to find the courage to invite change into our lives while accepting the things we cannot change.

Few of my integration practices involved creating a visual diary of imagery, archetypes, and dreams that arose during the three and a half months of treatment.

Journaling and reading. And later, fostering meaningful connections with others. Finding soulful physical activities that bring me joy. Accessing a spiritual framework of Buddhist Psychology from which to understand my suffering.

Also, I offered mindful attention to the parts of myself that were previously exiled, holding them in awareness without judgment, listening, and welcoming them into my being. And finally, for those of us with deep trauma, integration involves remembering and honouring the past and how far we have come most importantly, at times when we are triggered or in pain.

Question \#2: How important is Integration in the healing process, with or without psychedelics?

Integration is essential in the healing process. It is where most of the hard work is emotions, parts, and self. Without integration, one might expect to be magically healed in one session, but in practice, healing requires time, space, kindness, compassion, and working with what continues to emerge and what might continue to be painful. It also requires the development of life skills for growth in all areas of our life, including career, relationships, love, health, money, etc.

Question \#3: Have you any concerns regarding how Integration is, or is not, currently being integrated into the use of psychedelic substances?

I have several compassionate concerns. The MAPS phase two trials, for instance, offering only limited integration sessions (three 1.5 hour sessions post-psychedelic session). And participants then being let go with no road map for integration. 


\section{International Journal of Social Sciences and Management Review}

Volume: 03, Issue: 04 "July - August 2020"

ISSN 2582-0176

The larger MAPS community is excited about psychedelics and talks frequently about integration but may know very little about trauma and therefore mislead people who are suffering. I feel like integration is a word everyone is talking about, a buzzword used and at risk of losing meaning.

\section{Integration using the IFS model}

Integration is a core step in the Internal Family Systems (IFS) therapeutic model, which represents a new synthesis of two already-existing paradigms: systems thinking and the multiplicity of the mind. IFS brings concepts and methods from the structural, strategic, narrative, and Bowenian schools of family therapy to the world of sub personalities. This synthesis was the natural outcome that evolved after Richard Schwartz, PhD, a family therapist, began hearing from his clients about their inner lives. Once he was able to set aside his preconceived notions about therapy and the mind, and began to really listen to what his clients were saying, he heard repeatedly descriptions of what clients often called their "parts" -- the conflicted sub personalities that resided within them.

\section{Basic Assumptions of the IFS Model}

A. It is the nature of the mind to be subdivided into an indeterminate number of subpersonalities or parts.

B. Everyone has a Self, and the Self can and should lead the individual's internal system.

C. The non-extreme intention of each part is something positive for the individual. There are no "bad" parts, and the goal of therapy is not to eliminate parts but instead to help them find their non-extreme roles.

D. As we develop, our parts develop and form a complex system of interactions among themselves; therefore, systems theory can be applied to the internal system. When the system is reorganized, parts can change rapidly.

E. Changes in the internal system will affect changes in the external system and vice versa.

The implication of this assumption is that both the internal and external levels of system should be assessed.

\section{Overall Goals of IFS Therapy}

A. To achieve balance and harmony within the internal system

B. To differentiate and elevate the Self so it can be an effective leader in the system

C. When the Self is in the lead, the parts will provide input to the Self but will respect the leadership and ultimate decision making of the Self.

D. All parts will exist and lend talents that reflect their non-extreme intentions.

The IFS model incorporates the following steps to healing burdened parts - The 6 F's: 


\section{International Journal of Social Sciences and Management Review}

1. Finding a part to work with

2. Focusing on that part and where it may appear in or around the body

3. Fleshing out this part and identifying whether other parts are present

4. Asking the client how they Feel Toward the part

5. Befriending the part

6. Finding out the part's fears and concerns
B. Witnessing
C. Retrieval
D. Unburdening
E. Invitation
F. Integration
G. Acknowledging the parts/Gratitude

Once there is a Self-part connection the client can then witness the exile and/or a protective part's experience from a place of curiosity and compassion.

Conclusion

Integrating the psychedelic experience is essential for the achievement of clients' personal goals and growth. The intention to process the information gained during a psychedelic experience through the non-pathologizing, systems-oriented lens of the Internal Family Systems theoretical framework will help to understand how best to turn internal messages into real-world change.

Additional resource links:

htthttps://chacruna.net/integration-psychedelicsspirituality/ps://psychedelicstoday.teachable.com/p/psychedelic-integration-self-care https://www.psychedelicintegrationcoach.com/ https://www.medicinalmindfulness.org/psychedelic-integration-circle-boulder https://www.youtube.com/watch?v=E-5eFeICIf4 https://integration.maps.org/

Westrum, R., \& Dufrechou, J. (2019). The Psychedelics Integration Handbook. Buller, K. et al. (2019). Integration Workbook: Planting Seeds for Growth and Change 


\section{International Journal of Social Sciences and Management Review}

\section{REFERENCES}

Ackerman, S.J., Hilsenroth, M.J. (2003). A review of therapist characteristics and techniques positively impacting the therapeutic alliance. Clinical Psychological Review, 23, 1-33.

Lawlor, et al. (2017). Service user satisfaction with cognitive behavioural therapy for psychosis: Associations with therapy outcomes and perceptions of the therapist. British Journal of Clinical Psychology 56(1), 84-102.

Levitt, H., Butler, M., \& Hill, T. (2006). What clients find helpful in psychotherapy: Developing principles for facilitating moment-to-moment change. Journal of Counseling Psychology, 53(3), 314-324.

Littauer, H., Sexton, H., \& Wynn, R. (2005). Qualities clients wish for in their therapists. Nordic College of Caring Sciences, 19, 28-31.

Palmadottir, G. (2006). Client-therapist relationships: Experiences of occupational therapy clients in rehabilitation. British Journal of Occupational Therapy, 69(9), 394-401.

Sloshower, et al. (2020). Psilocybin-assisted therapy of major depressive disorder using Acceptance and Commitment Therapy as a therapeutic frame. Journal of Contextual Behavioral Science, 15, 12-19.

Ward, R., \& Morgan, N.L. (2017). Clients as Teachers: The 7 qualities most desired in a therapist. International Journal of Social Sciences, 3(2), 575-585. https://dx.doi.org/10.20319/pijss.2017.32.575585 